\title{
Die orthodoxe Kirchenmusik als ein bedeutendes Erbe von Byzanz und ihre moderne Rezeption im Westen am Beispiel des „Byzantinischen Kantorenchores München“
}

\author{
Konstantin NikOlakopoulos*
}

\begin{abstract}
The Orthodox Church Music as a Significant Heritage of Byzantium and its Modern Reception in the West: The Case of the 'Byzantinischer Kantorenchor München'
\end{abstract}

The Byzantine Music was created within the liturgical life of Orthodoxy and has been developed accordingly in the Eastern Church Worship. Together with the hymnography the Byzantine Music in Orthodoxy has from the beginning taken a central place, especially since there is absolutely no orthodox worship without psalmodic accompaniment. It is one of the most notable achievements in the Byzantine era, for which in the last decades also in Western Europe a great interest is awakened.

Keywords: Byzantine music, Orthodox worship, Hymnography, vocal music, Gregorian music, ecumenism, hymns, church modes, Ison, instrumental music free

\section{Einführendes}

Die Vielfalt der byzantinischen Errungenschaften in den verschiedenen Lebensbereichen wird besonders deutlich, wenn wir unsere Aufmerksamkeit auf zwei konkrete Sachbereiche fokussieren: auf die liturgisch-hymnographische und die kultisch-musikalische Produktion des über 1100 Jahre lang blühenden byzantinischen Reiches. Es handelt sich um zwei zentrale „göttliche Künste" der ostkirchlichen Tradition, die man im Rahmen des kultischen Vollzuges als die zwei Seiten einer Medaille bezeichnen könnte. In einer ganz besonderen Weise verbindet sich die Hymnographie, die keine eigenständige Größe im liturgischen Leben ist, mit der byzantinischen Kirchenmusik der orthodoxen Kirche. Die Wirkung des Wortes wird durch die Melodieführung der menschlichen Stimme unterstützt, die seinen Sinngehalt betont und erst recht lebendig macht. Die Hymnographie, welche die christliche Lehre dich-

* Konstantin Nikolakopoulos, Dr., Univ.-Professor für Biblische Theologie an der Ausbildungseinrichtung für Orthodoxe Theologie der Ludwig-Maximilians-Universität München (Deutschland), Ludwigstr. 29, D-80539 München; e-mail: Konstantin.Nikolakopoulos@ orththeol.uni-muenchen.de 
terisch formuliert, stellt eine Art „lyrische Theologie“ dar, während die Musik, die diese Lehre untermalt und zugleich zu ihrer Einprägung ins Herz der Gläubigen beiträgt, als „gesungene Theologie“ zu bezeichnen ist. In Byzanz wurde rund 1500 Jahre lang sowohl in Friedenszeiten wie auch in schrecklichen Notsituationen der geschriebene und der gesungene Lobpreis Gottes weiterentwickelt. Heute sind nur ca. 10\% des Hymnenbestandes in aktuellem liturgischem Gebrauch. Genau diese Texte umhüllt der byzantinische Kirchengesang musikalisch und verwandelt sie in rührendes Gebet, melismatische Danksagung oder flehentliche Bitte.

\section{Allgemeines zur byzantinischen Musik}

Die byzantinische Musik ist nichts anderes als die seit den frühchristlichen Jahrhunderten überlieferte musikalische Tradition des Griechentums, oder anders gesagt, des von der griechischen Kultur geprägten Christentums schlechthin. Diese Musik wurde innerhalb des liturgischen Lebens im oströmischen Reich „geboren“ und hat ihre Urgestalt in den ersten christlichen Jahren angenommen. Im Rahmen des Kultus fingen die Christen zuerst an, die Davidpsalmen zu singen, und danach wurden viele weitere gottesdienstliche Hymnentexte vertont. Allmählich deckte aber dieses musikalische System auch den Bereich der Volksmusik ab. Bis zum Ende des 19. Jh. wurde in Griechenland ausschließlich die byzantinische Melodik einerseits für den kirchlichen Gesang und andererseits für die profane Volksmusik gebraucht. Heutzutage steht sie besonders mit dem liturgischen Leben der orthodoxen Kirche, in dessen Rahmen sie intensiv und traditionsgemäß gepflegt wird, in enger Verbindung.

Hauptsächlich als liturgischer Gesang oder besser gesagt als „Psalmodie“ ist die byzantinische Musik nicht nur ein gemeinsames Gut aller Kirchen griechischer Sprache - Patriarchate von Konstantinopel, Alexandrien, Jerusalem, Kirchen von Griechenland und Zypern -, sondern auch aller Orthodoxen von Syrien und Palästina, die dem Patriarchat von Antiochien angehören und sich der arabischen Sprache bedienen. Schließlich ist die byzantinische Musik auch eine gemeinsame Tradition aller orthodoxen Balkanvölker, insbesondere der Rumänen und Bulgaren und zum großen Teil auch der Serben. Bei der russischen und finnischen Kirchenmusik sind große Abweichungen festzustellen, wobei aber noch Spuren des früheren Einflusses seitens der byzantinischen Musik zu finden sind. Weil die russische Musik seit dem 16. Jh. starke Einflüsse der polnischen, italienischen und deutschen Musik aufweist, ist sie in Westeuropa vertrauter und bekannter als die musikalische Tradition von Byzanz. 


\section{Musikalische Notation und handschriftliche Überlieferung}

Die Musik der Frühkirche hat sich in enger Parallelität mit der Hymnographie entwickelt. Nach orthodoxem Verständnis trägt der liturgische Gesang einen genuin geistigen Charakter, indem er sich für die persönliche Kommunikation des Gläubigen mit dem Göttlichen einsetzt. All dies wird durch die Tatsache deutlich gemacht, dass die alten Meloden, wie z. B. Romanos der Melode (6. Jh.) oder Johannes von Damaskus (8. Jh.), der als einer der ältesten Väter der byzantinischen Musik gilt, gleichzeitig berühmte Hymnographen und Heilige der Kirche sind. Die byzantinische musikalische Tradition wird bis heute gekennzeichnet durch ihre sorgfältige Überlieferung und ihre echte und traditionstreue Bewahrung von Generation zu Generation.

Die Tradition, welche die byzantinische Musik mit sich bringt, führt mindestens in die frühchristlichen Jahre zurück und hat ohne Zweifel auch mit der altgriechischen musikalischen Tradition vieles gemeinsam. Die erste Phase des schriftlichen kirchlichen Gesangs zeichnet sich durch die zwischen den Zeilen eines Textes hinzugefügten Neumen aus. Aber bereits im 9. Jh. waren die wichtigsten musikalischen Zeichen oder „Charaktere“ der kirchlichen Musik festgelegt und im 10. Jh. gab es ein vollständiges schriftliches System der byzantinischen Musik, die sog. Notation oder Parasemantik. Bemerkenswert ist die Tatsache, dass selbst auf dem Heiligen Berg Athos in den Jahren 950 bis 1100 ziemlich viele musikalische Handschriften verfasst wurden, wobei die erste datierte Handschrift, die von einem gewissen Anthimos geschrieben wurde, das Datum 31. Mai 1106 trägt.

Die Entwicklungsgeschichte dieser Kirchenmusik teilt sich in Anbetracht der Entstehung, Entfaltung und zuletzt Festlegung der heute gültigen Parasemantik in drei große Zeitabschnitte:

a) die frühbyzantinische Notation (9. - 12. Jh.)

b) die mittel- und spätbyzantinische Periode mit der alten synoptischen Notation (12. Jh. - 1814)

c) die Zeit der neuen analytischen Notation (1814 - heute).

Im Laufe der ganzen mittel- und spätbyzantinischen Zeit hat sich eine an Zeichen reichhaltige Parasemantik entwickelt, die aber allmählich nicht mehr übersichtlich sein konnte. Diese alte Notation umfasste um die 60 Zeichen/Charaktere; davon bezogen sich 15 Stimmzeichen ( $о \eta \mu \alpha \delta$ ó $\varphi \omega v a)$ auf Quantität (Ansteigen und Abfallen der Stimme) und ca. 45 große Hy-

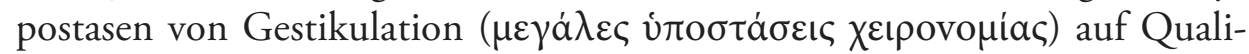
tät, künstliche Ausführung, Verzierung, Rhythmus etc. Die meisten Zeichen bargen eine vorbestimmte und in sich geschlossene Melodie, die deswegen 
synoptisch genannt wurde. Ein Schüler musste sich deshalb beim Erlernen dieser Notation auch die melodische Linie merken, die sich von jedem Zeichen und jeder Hypostase aus ergab. So entstand eine verwirrende Situation, die durch den Gebrauch von sowohl Quantitäts- als auch Qualitätszeichen hervorgerufen wurde.

Die „Interpretation“ dieser synoptischen Notation erfolgte im Jahre 1814, glücklicherweise sieben Jahre vor dem Beginn des griechischen Freiheitskampfes gegen die Türken. Die Musikreform wurde von drei ausgezeichneten Gelehrten jener Zeit vorgenommen, die durch den Namen „die drei Lehrer“ bekannt geworden sind:

- Chrysanthos von Madyta, Erzbischof von Dyrrhachion († 1843)

- Gregorios Lampadarios (†1822)

- Chourmouzios Chartophylax Hieropsaltes († 1840).

Die drei Lehrer schlossen mehrere ältere Zeichen von der neuen Methode aus, so dass von den Quantitätszeichen nur 10 (ison, oligon, petaste, kentemata, kentema, hypsele, apostrophos, hyporrhoe, elaphron, chamele) und von den zeitlosen Hypostasen nur 7 beibehalten wurden.

Außerdem erfolgte eine einheitliche Festlegung der verschiedenen Intervalle in der Quarte und den Tonleitern aller drei Genres, denen alle acht byzantinischen Kirchentöne zugeordnet werden (diatonisch, chromatisch, enharmonisch).

Ein weiterer Punkt der Musikreform von 1814 war auch die Ersetzung

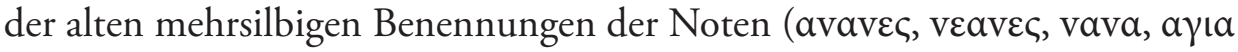

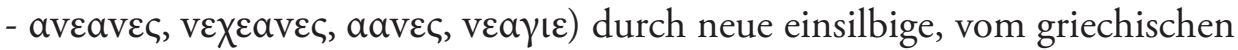
Alphabet herrührende Namen für die Töne ( $\pi \mathrm{A}$, Bov, $\Gamma \alpha, \Delta \mathrm{l}, \kappa \mathrm{E}, \mathrm{Z} \omega, \mathrm{vH})$.

Das neue analytische Parasemantiksystem von 1814 erwies sich als sehr übersichtlich und gebräuchlich und fand unter den Kirchenmusikern eine rasche Verbreitung. Die neue vereinfachte und überschaubare analytische Notation verursachte keinen Bruch mit der alten Gesangstradition, zumal die für die neue Methode ausgewählten Notenzeichen auch Bestandteile der alten gewesen sind. Die neue Notation hat hinsichtlich des technischen Aufbaus und der Zusammensetzung des byzantinischen Gesangs nichts Neues her-

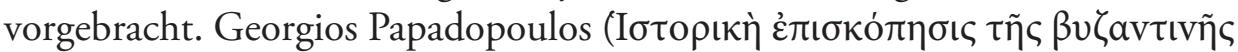

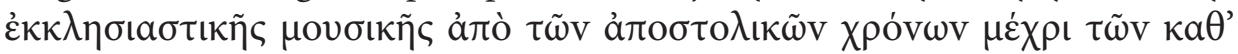

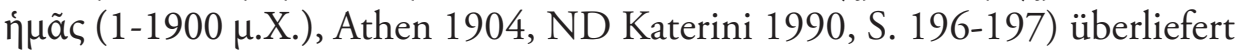
uns eine interessante Nachricht, die sich auf die Übergangsphase in den 20er und 30er Jahren des 19. Jh. bezieht: „Viele Kirchensänger im rechten Chor benutzten Bücher mit der Parasemantik von Petros dem Peloponnesios, während man im linken Chor - und umgekehrt - sich des neuen Notationssy- 
stems bediente; dennoch hat sich niemand beklagt, dass der andere die Melodien falsch ausführe."

Die mehr als 5500 bekannten Handschriften aus aller Welt zeigen sehr charakteristisch die ununterbrochene, aber sicherlich lebendige und vielfältige Entwicklung des byzantinischen Musiksystems. Alle bisher bekannten Meloden, es sind über 500, bewegen sich innerhalb ein und derselben musikalischen Kirchentradition. Man kann wohl mit Sicherheit behaupten, dass sowohl Kosmas von Majum (8. Jh.) als auch Johannes Kladas (15. Jh.) oder Konstantinos Pringos (20. Jh.) sich derselben byzantinischen Musik bedienen, deren gemeinsamer Anknüpfungspunkt innerhalb dieser großen zeitlichen Distanz hauptsächlich die mündliche Überlieferung ist. Daher wird bis heute berechtigterweise die Bezeichnung „byzantinische Musik"verwendet.

\section{Merkmale der byzantinischen Musik}

Die liturgische Musik war und ist schon immer reine Vokalmusik. Die Abwesenheit von Musikinstrumenten bzw. Kirchenorgeln ist kein Mangel, sondern eine theologisch und anthropologisch begründete Selbstverständlichkeit. In der orthodoxen Tradition gilt die von Gott geschenkte menschliche Stimme als das natürlichste und perfekteste Instrument zum Ausdruck des „Wortes" und darüber hinaus der Musik, welche den geschriebenen Text musikalisch wiedergibt. Die reichhaltige Hymnographie der Kirche bezweckt das unmittelbare Gespräch der Gläubigen mit Gott und setzt die unmittelbare Beziehung zwischen beiden voraus. Zugunsten dieser persönlichen Beziehung setzt also der Mensch seine natürliche Stimme im Rahmen der Gottesdienste ein, denn allein sie ist in der Lage, sich direkt an Gott zu wenden und zu bitten und darüber hinaus die geistige Atmosphäre der Gemeinde gebührend auszudrücken. In diesem Zusammenhang wird die menschliche Stimme hochgeschätzt, indem allein sie Musik und Sprache kombinieren und hervorbringen kann.

Es gab und es gibt zwar noch heute konkrete „byzantinische“ Instrumente; sie wurden und werden aber immer noch nur außerhalb des kirchlichen Raumes verwendet, entweder als Hilfe und Begleiter zum Erlernen der byzantinischen Musik, oder als profane Instrumente im Rahmen der Volksmusik. In allen alten Handschriften bedient sich auch die Volksmusik sowieso der byzantinischen Notation.

In der Regel ist die im Westen vorherrschende Kirchenorgel im liturgischen Bereich der Orthodoxie unbekannt, wobei das Abendland die Orgel allerdings als profanes Instrument - im frühen Mittelalter von Byzanz übernahm. Im Jahre 757 nämlich schenkte der byzantinische Kaiser Konstantinos 
V. dem Franken Pipin eine Orgel und diese wurde dann in der lateinischen Kirche zum Kircheninstrument schlechthin.

Das Fehlen der Musikinstrumente in der orthodoxen Kirche und logischerweise die Festlegung der Kirchenmusik als reine Vokalmusik stützen sich ebenfalls auf eine ununterbrochene patristische Tradition und folgen anhaltend und treu der Entwicklung der byzantinischen Musiktradition. Wie es sich aus diesbezüglichen Reden vieler Kirchenväter ergibt, z. B. von Eusebios von Kaisareia, von Gregor dem Theologen oder auch von Isidor von Pelusion, wurden alle Instrumente, die von der Kirche nicht als solche verurteilt werden, von dem Gottesdienst ausgeschlossen, damit der Unterschied sowohl zwischen christlichem und jüdischem Kultus, der manchmal Instrumente einsetzte, als auch zwischen „Orthodoxie“ und häretischen Sekten, die zum Gewinn naiver Gläubigen profane Instrumente verwendeten, verdeutlicht und offenbart werde.

Erwähnenswert ist in diesem Zusammenhang die Tatsache, dass viele alttestamentliche Verse, die die wichtigsten Musikinstrumente dieser Zeit und dazu auch den Tanz in Verbindung mit dem Lobpreisen Gottes bringen, in den liturgischen Texten der Orthodoxie zwar vorhanden sind, nicht aber wortwörtlich, sondern im Geist und Licht des Neuen Testaments verstanden und erläutert werden. Den obengenannten Versen kommen, entsprechend dem spirituellen Charakter des orthodoxen Kultus, ebenso mehr eine symbolische Bedeutung und ein geistiger Sinn zu.

Neben diesen theologischen Überlegungen darf hierbei noch eine Tatsache erwähnt werden, die ihre Gültigkeit dem musikwissenschaftlichen Bereich entnimmt und den Ausschluss aller Instrumente unterstützen vermag: In der byzantinischen Musik ist eine verschiedenartige Unterteilung des Tonabstandes in größere und kleinere Intervalle möglich, so dass dieser Intervallreichtum der byzantinischen Gammen mit den profanen Instrumenten, die nur über die Hauptintervalle (Ton und Halbton) der europäischen Musik verfügen, nicht authentisch wiedergegeben werden kann. Jeder der acht Kirchentöne der byzantinischen Musik, der sich durch seine eigenen Zwischentöne und Verschleifungen auszeichnet, würde etwa durch seine angebliche Anwendung im europäischen Musiksystem nicht authentisch wiedergegeben. Die byzantinische Musik besitzt eine eigene musikalische Farbe und einen konkreten Charakter, welche für die Mitwirkung der Psalmodie bei den verschiedenen Gottesdiensten eine grundlegende Rolle spielen.

Ein weiteres, sehr bedeutendes Merkmal der liturgischen Musik in der orthodoxen Kirche bildet ihre seit den ersten Jahrhunderten belegte Einstimmigkeit. Das monophonische Melos, d. h. die kirchliche Psalmodie, die keine 
Polyphonie westlicher Art kennt, wird ausschließlich von einem Grundton, dem sog. "Isokratema“ oder "Ison" unterstützt bzw. getragen, wodurch ein falscher Eindruck der Mehrstimmigkeit entsteht. In Wirklichkeit bildet aber dieser Grundton, der einfach die Basis des jeweils zu singenden Kirchentones ist, keine eigenständige Melodie, sondern eine Begleitung der Melodie, wie

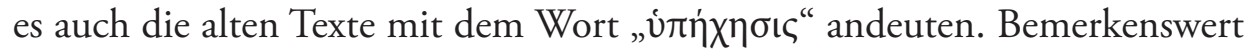
in diesem Zusammenhang ist folgende exegetische Ausführung von Johannes

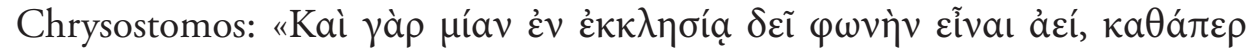

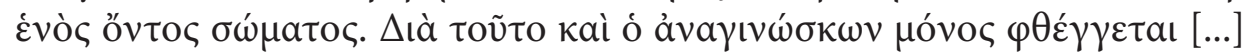

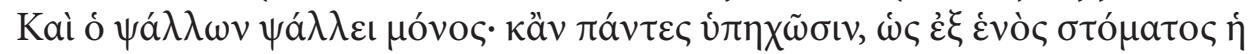

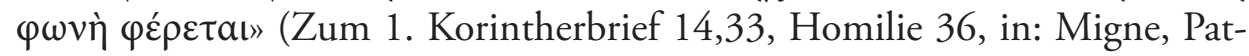
rologia Graeca 61,315).

\section{Praktische Ausführung der byzantinischen Musik}

Nachdem die Liedkompositionen eine enorme Entfaltung und Ausdehnung erfahren hatten, war es notwendig, dass der Gesang der meisten Hymnen nicht mehr vom ganzen Volk, sondern von ausgebildeten Psalten/ Kantoren und Chören übernommen wurde. Beim Vollzug der orthodoxen Gottesdienste wirken im musikalischen Bereich also sowohl Kantoren als auch Lektoren mit.

Die Ersten singen abwechselnd die Mehrheit der Hymnen oder leiten einen am Gottesdienst beteiligten Chor. Dabei könnte der zentrale Kirchensänger nur dirigieren und als Mitglied des Chores die einstimmige Melodie mitsingen oder aber auch konkrete, vorherbestimmte Hymnenabschnitte, die eine besondere Ausführungsweise mit Verschleifungen erfordern, nur mit Isokratema-Begleitung solo singen. Traditionsgemäß sind fast alle Kirchensänger Männer, das heißt aber nicht, dass der Gesang den Frauen verboten wird. Es gibt erwähnenswerte Ausnahmen von weiblichen Kantoren und nicht zuletzt übernehmen in den Frauenklöstern ausschließlich Nonnen das Singen.

Die Lektoren übernehmen in der Regel das Vortragen oder Rezitieren von gewissen Psalmen und Gebeten. In den Klöstern und in manchen Stadtgemeinden führen sie aber auch das sogenannte "Kanonarchema“ aus. Dabei handelt es sich um das schrittweise Rezitieren der Texteinheiten eines Hymnus, als ob er vom Kanonarches dem Kantor diktiert wird, um dann von den Sängern oder Chören melodisch gesungen zu werden. Durch dieses rezitative Vortragen der Texte, das sich musikalisch auf der Basisnote des jeweils zu singenden Kirchentones bewegen muss, kann das gläubige Volk die Einzelnwörter und den gesamten Inhalt des Hymnus deutlich und somit verständlich hören. 


\section{Der Charakter bzw. das Ethos der byzantinischen Musik}

Die byzantinische Musik wirkt mehr oder weniger innerhalb eines sehr stark von Gefühlen geprägten Bereiches. Allerdings ist es unzweifelbar, dass Musik schlechthin als Ausdruck der tiefsten Leidenschaften und der edelsten Gefühle und Sehnsüchte der Menschheit gilt. Aus einem anderen Blickwinkel wird der Musik aber zugleich der Charakter der Ruhe und der Leidenschaftslosigkeit zugeschrieben, wobei sie auch zur seelischen bzw. körperlichen Heilung des Menschen beizutragen weiß. Diese bekannten Vorstellungen der Antike, welche mehr oder minder bis heute vertreten werden, bilden keine Neuheit im Rahmen der byzantinischen Musik. Es ist für die Kirchenmusik der Orthodoxie nämlich charakteristisch, dass sie alle obengenannten Elemente beinhaltet und sie entschieden zum Ausdruck bringt.

Durch die Musik kann man einerseits die christliche Lehre und die theologischen und erlösenden Wahrheiten im Gedächtnis und im Herzen festhalten und andererseits seinen Glauben samt seinen persönlichen Gefühlen ausdrücken. Der Psaltes, aber auch jeder Mensch, der tief in den Sinn der Musik eingeweiht ist, kann durch das Singen eine Vielfalt von Gefühlen empfinden und zugleich ausdrücken. Man könnte, grob eingeteilt, zwei Grundelemente im orthodoxen Kultus anführen: das des Gebetes und das des Lobes. Auf diese Weise ist die byzantinische Musik aufgefordert, auf der einen Seite das Leid und die Trübsal des Sünders und seine Furcht vor dem Jüngsten Gericht und auf der anderen Seite den Jubel, die Fröhlichkeit und die Freude über die Auferstehung bzw. die Erlösung zum Ausdruck zu bringen. Diese zwei Elemente, welche sich zu der in der östlichen Theologie bekannten

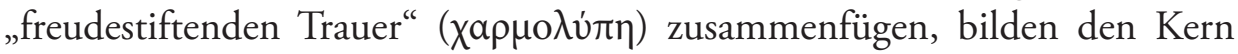
des orthodoxen Kultus.

Der byzantinische Gesang ist durch seinen technischen Aufbau dazu befähigt, die gebührende liturgische Atmosphäre vollkommen wiederzugeben. Diese wahre und wirkliche Kunst beinhaltet nämlich die Verschiedenheit der acht Kirchentöne, von denen jeder einen einzigartigen Charakter aufzeigt, mit denen sie eine Vielfalt von Gefühlen ausdrückt. Erwähnenswert ist in diesem Zusammenhang, dass alle Theoretiker und Väter der byzantinischen Musik das Wort Ethos benutzen, wenn sie vom Charakter jedes Tones reden; ein Wort, das sicherlich viel tiefer und viel ausdrucksvoller ist als die Bezeichnung „Charakter“.

Die byzantinische Musik wird mit Recht als die „Bekleidung des Wortes" bezeichnet, weil sie dazu dient, dass der Sinn der Texte, der hinter den Buchstaben verborgen ist, lebhaft und entschieden zum Ausdruck gebracht 
wird. Es ist eines der wichtigsten Prinzipien des byzantinischen Melos, jedes Wort eines Hymnus treffend zu betonen und seinen eigentlichen Sinn absolut ernst zu nehmen. Je nach dem inhaltlichen Stil des jeweiligen Hymnus wird derjenige Kirchenton angewandt, der den beabsichtigten Ausdruck und die zu äußernden Gefühle am besten wiedergeben kann. Es ist z. B. kein Zufall, dass fast alle Hymnen, die das Thema Buße bzw. Reue behandeln, innerhalb des diatonischen Genres und vorzugsweise im achten, dem Nebenton des vierten Kirchentones gesungen werden; ein Ton, der beim Singen am eindrucksvollsten Demut und Rührung auszudrücken weiß.

$\mathrm{Zu}$ erwähnen ist hier ebenso die Tatsache, dass die Genres innerhalb des byzantinischen Musiksystems und je nach angezielter Bedeutung des Textes sehr häufig kombiniert werden. Ein Hymnus, der das christliche Leben eines Heiligen beschreibt und preist, wird z. B. wegen des fröhlichen Charakters im diatonischen Genre gesungen. Angenommen aber, dass im Verlauf des Textes die Formulierung vorkommt „er hat den Weg der Sünder nicht beschritten“, dann ist es möglich und wahrscheinlich, dass das Wort „Sünder“ durch eine außerordentliche Zeichenänderung nicht mehr im diatonischen, sondern im chromatischen Genre gesungen wird. Dass ein Melode dies so handhabt, wird dadurch erklärt, dass ausschließlich das chromatische Genre dazu befähigt ist, die Leidenschaft und die Trauer musikalisch hervorragend wiederzugeben.

Ein zweites Beispiel könnte die durch die Musik geschaffene Emphasis (Nachdruck) des Textes vielleicht noch deutlicher zeigen. Ein Doxastikon, das den Menschen zu Gerechtigkeit, Almosen und Demut aufruft, endet mit der Phrase des Hymnographen „auf diese Weise wird Gott uns die himmlischen anstelle der irdischen Güter gewähren“. Um die unterschiedliche Bedeutung der beiden Dimensionen aber am besten zu veranschaulichen, vertont der Melode das Wort „himmlisch“ mit höheren Tönen der oberen Oktaphonie und lässt das Wort „irdisch“ durch charakteristische Töne der unteren Gamme eindrucksvoll tief klingen. Dadurch wird der Text ausdrucksvoll, und die einzelnen theologischen Wahrheiten werden für das gläubige Volk deskriptiv verlebendigt. Im Allgemeinen kann man sagen, dass die byzantinische Musik als Begleiterin und Interpretin der Hymnographie dem kultischen Leben der Kirche wirklich einen unschätzbaren Dienst erweist.

\section{Die Pflege und Präsentation der byzantinischen Musik im Westen am Beispiel des „Byzantinischen Kantorenchores München“}

Der Byzantinische Kantorenchor ist die besondere musikalische Ausdrucksweise des „Vereins für Byzantinische Musik München e.V.“, der am 3. Oktober 2003 offiziell gegründet wurde. Hauptgründungsinitiator war der 
Studiengang für orthodoxe Theologie an der Ludwig-Maximilians-Universität München, aus dem mehrere Professoren, Dozenten und Studierende im byzantinischen Chor wirken. Als wichtiges Motto des Chores, der seit 2005 von Prof. Dr. Konstantin Nikolakopoulos geleitet wird, wäre die kulturelle Begegnung zu nennen. Die Verantwortlichen des Vereins für Byzantinische Musik und des Byzantinischen Kantorenchores engagieren sich für die Förderung und Pflege der griechisch-byzantinischen Kirchenmusik und Tradition, den Kulturaustausch zwischen deutschen und griechischen Mitbürgern sowie die Förderung der orthodoxen musikalischen Kultur in ihrer Begegnung mit anderen europäischen Kulturkreisen. Diese Ziele will der Kantorenchor erreichen durch:

- die sorgfältige Pflege und traditionstreue Bekanntmachung und Präsentation der byzantinischen Musik der orthodoxen Kirche im westeuropäischen Kulturbereich durch Vorträge, Publikationen, liturgische Events oder Konzerte

- die musikalische Bildung und Erziehung von Interessierten

- die ungefähre Musikübertragung aus dem byzantinischen Notationssystem (Neumenschrift) in das westeuropäische Notensystem in Anlehnung an die byzantinische Musiktradition (man kann nicht von einem hundertprozentigen Erfolg sprechen - die akustischen Ergebnisse klingen relativ byzantinisch)

- die Anpassung der ins Deutsche übersetzten Hymnentexte an die byzantinische Gesangsweise für den liturgisch-gottesdienstlichen Gebrauch im deutschsprachigen Raum

- die Zusammenarbeit mit anderen Musikvereinen sowie mit einzelnen Persönlichkeiten aus der Musik im In- und Ausland.

\section{Eine kulturelle Begegnung zwischen byzantinischer Musik und Gregorianik}

Um sich ein authentisches Bild über die Wahrnehmung der byzantinischen Musik in Westeuropa machen zu können, möchte ich nicht auf die zahlreichen Aufführungen und Konzerte des Byzantinischen Kantorenchores, sondern auf ein besonderes kulturelles Ereignis als wichtiges Beispiel Bezug nehmen. Es handelt sich um ein gemeinsames Konzert zweier Chöre, das am 12. Juni sowie am 3. Juli 2010 stattfand und genau der oben beschriebenen Begegnung der östlichen und westlichen Musiktradition des Christentums verpflichtet war. Als Ausführungsort wurde keine andere musikalische oder spirituelle Stätte als die traditionsreiche Benediktinerabtei Niederaltaich ausgewählt. Das Benediktinerkloster Niederaltaich ist ein der Überlieferung nach im Jahr 741 von Herzog Odilo von Bayern gegründetes, in der Gemeinde Niederalteich an der Donau 
liegendes katholisches Kloster, das dem heiligen St. Mauritius geweiht ist. Dort leben östliche und westliche Tradition seit Jahrzehnten harmonisch zusammen. In diesem besonderen Benediktinerkloster wird seit mehreren Jahren auch die östliche orthodoxe Spiritualität in all ihren Formen, auch durch die Musik, treu studiert, gepflegt und erlebt.

Das Konzert offenbarte also noch einen weiteren wichtigen Aspekt der ökumenischen Annäherung und der eindrucksvollen spirituellen Gemeinsamkeiten der römisch-katholischen und der orthodoxen Tradition. Beteiligt daran waren zwei Chöre, die diese beiden Traditionen genuin repräsentieren. Für die orthodox-byzantinische Seite trat der Byzantinische Kantorenchor des Vereins für Byzantinische Musik München e.V. und für die katholisch-gregorianische Seite der Chor der Pueri Cantores Altahensis der Abtei Niederaltaich auf. Letzterer ist der bekannte Knabenchor des Klosters, der unter der Leitung von P. Romanos Werner OSB die jahrhundertelange Tradition der Sänger studiert und durch sein musikalisches Engagement den Gesang der Mönche von Niederaltaich beim Vollzug der verschiedenen Gottesdienste unterstützt.

Die Hintergrundinformationen auf der Außenseite des gedruckten Programms haben genau den Hauptzweck der Veranstaltung wiedergegeben: „Seit Anbeginn des Christentums ist an zabllosen Orten eine Fülle von Hymnen und Gesängen entstanden. Aufbauend auf das Erbe der Synagogenliturgie haben die Christen sehr bald begonnen, eigene Hymnen und Liedgut - inspiriert durch den Heiligen Geist - neu zu schöpfen. Schon die Schriften des Neuen Testamentes geben Zeugnis von diesem Schaffen... Im Laufe der Jahrhunderte haben sich, durch die Gegebenheit verschiedener kultureller Einflüsse, in allen Teilkirchen eigene musikalische Traditionen herausgebildet. Die Stimme der Völker hat dem Wort Gottes in der jeweiligen Verkündigung einen je eigenen Klang verliehen und damit zur Herausbildung der verschiedenen christlichen Musiktraditionen geführt. Durch interkulturellen Austausch und andere Momente der Kirchengeschichte sind sich diese Traditionen immer wieder begegnet und haben sich gegenseitig beeinflusst. "

Das verbindende Element des besonderen „ökumenischen“ Musikkonzerts der beiden Chöre, die ihrerseits zwei verschiedene musikalische Traditionen des Christentums (östlich-byzantinische und westlich-gregorianische) echt und treu repräsentierten, war die gemeinsame Hymnographie der beiden Traditionen, die auf die eine ungeteilte Kirche zurückzuführen ist. Anhand von hymnologischen Texten, die in den beiden großen christlichen Kirchen von Anfang an im liturgischen Gebrauch zu finden sind, kam der jeweilige musikalische Umgang zum Vorschein. Aus orthodoxer Perspektive handelte es sich um zentrale Hymnen, Lieder und Akklamationen aus dem täglichen Gebet und den Abend- und Morgengottesdiensten sowie der Göttlichen Liturgie. 
Am Anfang sangen wir das bekannte Gebet zum Heiligen Geist „Himmlischer König“, das in der lateinischen Tradition dem Hymnus „Veni Creator Spiritus" entspricht. In der orthodoxen Tradition bildet dieser Hymnus, der hymnologisch vom „hohenpriesterlichen Gebet“ Jesu Christi in den Kapiteln 14-16 des Johannesevangeliums inspiriert ist (hier: Joh 15,26 f.), einen der beliebtesten Gebetstexte zum Heiligen Geist. Er wird bei der Eröffnung jedes privaten Gebets und eines jeden Abend- und Morgengottesdienstes wie auch insbesondere während der Pfingsttage rezitiert bzw. melismatisch wiedergegeben. Die beiden Chöre haben dann den bekannten liturgischen Zuruf „Kyrie eleison“ in ihren verschiedenen musikalischen Weisen vorgestellt. An dritter Stelle wurde die sogenannte „Große Doxologie“ (das „Gloria“), die ihren Ursprung im Engelgesang des Lukasevangeliums ( $\operatorname{Lk} 2,14)$ und ihre liturgische Ausgestaltung in den ,apostolischen Konstitutionen" hat, gesungen.

Unsere Chöre haben dann einen zentralen festen Teil jeder orthodoxen Göttlichen Liturgie, das berühmte und beliebte „Trishagion“ („Heiliger Gott, Heiliger Starker, Heiliger Unsterblicher, erbarme Dich unser“) in traditionellen Melodien vorgestellt. Im römischen Ritus wird es - in griechischer und lateinischer Sprache mehrmals wiederholt - in den „Improperien" bei der Kreuzverehrung am Karfreitag gesungen. In der orthodoxen Göttlichen Liturgie wird anstelle des Trishagion an den großen Festen zu Ehren des lebenspendenden Kreuzes der Hymnus „Dein Kreuz verehren wir, Gebieter..." rezitiert, den wir in einer alten Melodie des 2. byzantinischen Kirchentones präsentiert haben. Die Pueri Cantores Altahensis sangen das „Crucem tuam adoramus“ aus der Kreuzverehrung in einer mehrstimmigen Weise aus einer alten Handschrift des Benediktinerklosters auf dem Montecassino. Nach zwei sehr melismatischen Psalmen-Vertonungen, nämlich Ps 50 (51) für die westliche und 67 (68) für die östliche Tradition, sangen die beiden Chöre einen weiteren gemeinsamen Hymnus, der in der byzantinischen Liturgie anstelle des Trishagion bei den großen Herrenfesten feierlich erklingt und einer zentralen theologischen Formulierung des Apostels Paulus (Gal 3,27) entnommen ist: „Die auf Christus ihr seid getauft, habt Christus angezogen. "In der römischen Tradition ist dieser Vers der Kommunionsgesang am Fest der Taufe Christi. Die Sängerknaben sangen ihn im einstimmigen gregorianischen Choral, die zugehörigen Verse als mehrstimmige Falsibordoni. Das Konzert wurde mit zwei wichtigen gemeinsamen Hymnen aus dem Abendgottesdienst abgeschlossen: dem berühmten Hymnus "Heiteres Licht" (westlich in einer englischen Fassung) sowie dem bekannten Loblied an die Gottesgebärerin "Theotoke Parthene", das dem westlichen „Ave Maria“ entspricht. 


\section{Nachwort}

Es ist eine Tatsache, dass die byzantinische Musik innerhalb des liturgischen Lebens der Orthodoxie „geboren“ und im Rahmen des ostkirchlichen Kultus entsprechend gestaltet wurde. Das, was gemäß dem orthodoxen Verständnis wichtig ist, ist die Verwirklichung der angestrebten Beziehung des Menschen zu Gott. Die verschiedenen Charaktere aller acht Kirchentöne tragen gewiss dazu bei, dass der Mensch, der sich vor Gott stumm und unwürdig fühlt, Ihn zu preisen, die Möglichkeit hat, auch durch die Musik seine Gefühle zu äußern und darzubringen.

Die Musik ist ein von Beginn der christlichen Kirche her in Anspruch genommenes Mittel im Kultus, gleich wie die Hymnendichtung, die Ikonen, der Weihrauch, die priesterlichen Gewänder und Ähnliches. In der Orthodoxie nahm die byzantinische Musik seit jeher einen zentralen Platz ein, zumal es absolut keinen orthodoxen Gottesdienst ohne psalmodische Begleitung gibt. Zugleich hat diese Musik den profanen weltlichen Bereich des oströmischen Reiches ebenfalls geprägt, indem sie als Volksmusik alle wichtigen Situationen des Lebens, wie Geburt, Liebe, Freude, Hochzeit, Emigration, Freundschaft oder Tod begleitete und musikalisch zum Ausdruck brachte. Es handelt sich also tatsächlich um eine der erwähnenswertesten Errungenschaften in der byzantinischen Ära, deren Pflege und Weiterführung bis heute als eine wertvolle Aufgabe und Verantwortung für die Orthodoxen zu betrachten ist. 